\title{
Editorial
}

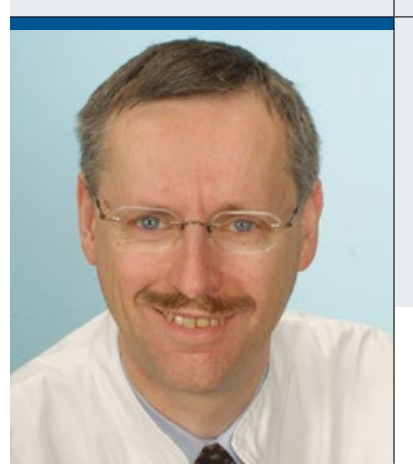

"Die Lockerung der Zielblutdruckwerte wird sich sicher

wieder umkehren."

\section{W. Zidek}

Med. Klinik IV

Charité - Universitätsmedizin Berlin

\section{Ungelöste Kontroversen in der Hypertonie}

n der Hypertonietherapie blicken wir auf eine Reihe ungelöster Kontroversen: Ausgelöst durch die SPRINT-Studie gab es die Debatte um die Zielblutdruckwerte [1], die gezeigt hat, dass die noch gültige, eine Zeit zurückliegende Lockerung der Zielblutdruckwerte offenbar kein Fortschritt in der Sache gewesen ist. Die Tendenz wird sich ohne Zweifel wieder stärker in Richtung einer schärferen Blutdruckeinstellung bewegen. Allerdings wurden auch die Grenzen der Blutdrucksenkung aufgezeigt: HOPE-3 hat gezeigt, dass die "präventive“ Behandlung von Blutdruckwerten im oberen Normbereich bei Patienten mit mittlerem kardiovaskulären Risiko keine signifikanten Vorteile bringt [2].

Das Phänomen der J-Kurve gehört ebenfalls zu den ungelösten Kontroversen. Die grundsätzliche Akzeptanz einer J-Kurve ist nicht das Problem. Alle, die Grundlagen der menschlichen Physiologie kennen, wissen, dass es eine J-Kurve geben muss... Es geht vielmehr darum, ob der Nadir der J-Kurve in einem Bereich liegt, der häufiger therapeutische Konsequenzen mit sich bringt. In der kürzlich publizierten CLARIFY-Studie [3] sehen wir einen Nadir kardiovaskulärer Ereignisse für KHK-Patienten bei 120/70 mmHg, während die INVEST-Studie, eine der größeren Studien, die das J-Kurven-Phänomen bekannter gemacht haben, den Nadir zwischen 135$145 \mathrm{mmHg}$ systolisch und 60-90 mmHg diastolisch zeigte [4]. Die neue Studienlage relativiert also die Bedeutung der J-Kurve: Wir würden ohnehin eine Blutdrucksenkung deutlich unter $120 / 70 \mathrm{mmHg}$ in den seltensten Fällen anstreben.

In 2016 ist eine weitere interessante Studie zum Thema der J-Kurve publiziert worden [5]. Sie enthält Daten, die ein differenzierteres Bild vermitteln. Die Autoren haben an 11.565 Probanden über 21 Jahre den Zusammenhang zwischen diastolischem Blutdruck und Myokardschädigung untersucht. Ein diastolischer Blutdruck war mit erhöhter Inzidenz einer
KHK oder eines erhöhten Troponinwertes nur dann assoziiert, wenn der systolische Blutdruck gleichzeitig $\geq 120 \mathrm{mmHg}$ war. Es besteht ein Zusammenhang zwischen dem Risiko eines erhöhten Troponin-Wertes und dem diastolischen Blutdruck in Abhängigkeit von der systolischen Blutdruckhöhe. Dies lässt vermuten, dass die erhöhte Blutdruckamplitude (Pulsdruck) der entscheidende Faktor ist, der die kardiale Prognose verschlechtert. Dies würde die Studienergebnisse plausibel machen, weil dann der erniedrigte diastolische Druck bei gleichzeitig niedrigem systolischen Druck eben unschädlich wäre.

Auch der Stellenwert der invasiven Hypertonietherapie ist nach wie vor ein ungelöstes Problem. Die vielerorts unkritische Anwendung speziell der renalen Denervation schien das technisch Machbare zum Maßstab der Indikation zu machen. Da war eine Umkehr ins Gegenteil nicht verwunderlich. Es wird voraussichtlich noch geraume Zeit brauchen, bis der angemessene Stellenwert der invasiven Therapie der resistenten Hypertonie definiert ist.

Eine weitere ungelöste Kontroverse betrifft das rationale Vorgehen bei resistenter Hypertonie: PATHWAY-2 [6] hat Daten gezeigt, die für einen routinemäßigen Einsatz von Spironolacton bei resistenter Hypertonie sprechen. Es gibt aber eine Reihe von Publikationen in weniger prominenten Zeitschriften, die bessere Ergebnisse mit einem individualisierten Ansatz bei resistenter Hypertonie zeigen.

Die im Schwerpunkt dargestellten Themen hängen mit einer dieser Kontroversen zusammen.

Ich wünsche den Lesern viel Vergnügen mit dem Themenschwerpunkt und hoffe, dass wir in 2017 der Lösung dieser Kontroversen näher kommen.

Ihr Walter Zidek

\section{Literatur}

www.springermedizin.de/cardiovasc 


\section{Literatur}

1. Group SR, Wright JT Jr., Williamson JD et al. A Randomized Trial of Intensive versus Standard Blood-Pressure Control. N Engl J Med. 2015:373:2103-16

2. Lonn EM, Bosch J, Lopez-Jaramillo P et al. Blood-Pressure Lowering in IntermediateRisk Persons without Cardiovascular Disease. N Engl J Med. 2016;374:2009-20

3. Vidal-Petiot E, Ford I, Greenlaw $\mathrm{N}$ et al. Cardiovascular event rates and mortality according to achieved systolic and diastolic blood pressure in patients with stable coronary artery disease: an international cohort study. Lancet. 2016;388:2142-52

4. Bavry AA, Anderson RD, Gong Y et al. Outcomes Among hypertensive patients with concomitant peripheral and coronary artery disease: findings from the INternational VErapamil-SR/Trandolapril STudy. Hypertension. 2010:55:48-53

5. McEvoy JW, Chen Y, Rawlings A et al. Diastolic Blood Pressure, Subclinical Myocardial Damage, and Cardiac Events: Implications for Blood Pressure Control. J Am Coll Cardiol. 2016;68:1713-22

6. Williams B, MacDonald TM, Morant S et al. Spironolactone versus placebo, bisoprolol, and doxazosin to determine the optimal treatment for drug-resistant hypertension (PATHWAY-2): a randomised, double-blind, crossover trial. Lancet. 2015;386:2059-68 